\title{
Genetic aspects of internet-dependence in teenagers
}

\author{
Marina Smolnikova \\ Laboratory of cell-molecular physiology and pathology \\ Scientific Research Institute of Medical Problems of the North \\ FRC KSC SB RAS: Krasnoyarsk, Russia \\ smarinv@yandex.ru
}

\begin{abstract}
The rapid emergence and spread of Internet addiction in adolescent populations, combined with a rapid change in consumed content due to mobile access availability and the new access devices, are new challenges for classical psychology and fundamental medicine that require urgent solutions. The presence of the genetic component of Internet addiction was convincingly shown in different populations, but to date the specific genes involved in the mechanisms of such heritability have not been well identified. The aim of the study was to investigate the role of candidate neurotransmitters and to perform a population analysis of candidate genes polymorphisms for the Internet addiction formation. As a result of the analysis, 7 Internet-dependent adolescents and 39 adolescents with a tendency to Internet-dependent behavior were identified. The frequencies of genotype distribution in the population sampling of Caucasians $(\mathbf{n}=\mathbf{3 0 2})$ were obtained: * CC 59.6\%, * CT 40.4\%, * TT 0\% (rs1800497 DRD2); * AA $23.8 \%$, AG 52.4\%, * GG 23.8\% (rs4680 COMT); * CC 37.4\%, * CG 46.0\%, * GG 16.6\% (rs2229910 NTRK3). The results will help to open new perspectives in assessing the fundamental neurobiological causes for the Internet addiction and the personalization of therapeutic approaches in Internet dependent adolescents.
\end{abstract}

Keywords - adolescents, Internet addiction, gene polymorphism

\section{Introduction}

Internet addiction (IA) is a relatively new psychological phenomenon having signs of a social epidemic, specifically attributed to vulnerable groups (adolescents and young adults) in some regions (South Korea, China, Japan). The prevalence of IA among adolescents varies depending on the ethnosocial groups studied and the diagnostic criteria used, from $1 \%$ to $18 \%$ [1]. In Europe, therefore, the prevalence of IA among teenagers is $1-11 \%$, on average $4.4 \%$, in the USA, the prevalence of IA in the total sampling is $0.3-8.1 \%$. However, the prevalence of IA among teenagers and young adults is significantly higher - 9-37.9\% in Asian countries [2].

The genetic component of developing Internet addiction was shown by twin studies using various populations, however, to date, the specific genes involved in the mechanisms of such heritability are not exactly identified. Pilot studies verified some polymorphic sites of five candidate genes: rs1800497 (dopamine D2 receptor (DRD2) gene, Taq1A1 allele) and rs4680 (methionine variant of dopamine degradation enzyme catecholamine-o-methyltransferase (COMT) gene), and the association of minor alleles associated with low dopamine production (rs4680) and a low number of dopamine receptors in the prefrontal cortex (rs1800497) with pathological addiction to Internet games in adolescents in South Korea was shown to occur [3]. An exome analysis including a study of 83 polymorphic sites revealed a statistically significant association only with rs2229910 (neurotrophic tyrosine kinase receptor type 3 (NTRK3) gene), perhaps also associated with anxiety, panic, depression and other disorders [4].

\author{
Sergey Tereshchenko \\ Clinical department somatic and mental health of children \\ Scientific Research Institute of Medical Problems of the North \\ FRC KSC SB RAS, Krasnoyarsk, Russia \\ legise@mail.ru
}

Thus, the emergence and spread of Internet addiction in adolescent, combined with the rapid change in consumable content due to the general availability of mobile access to the network and appearing some new access devices, pose for fundamental medicine some new challenges to be studied. This is particularly true because of the established serious comorbidity of IA (depression, anxiety, suicidal behavior, unmotivated aggression, as well as psychosomatic diseases). An analysis of ethnogeographic differences of Internet addiction, while taking into account the ethnic differences in the prevalence of genotypic characteristics of populations seems to be relevant and that is not sufficiently explored in modern neurogenetics of addiction in adolescents, especially in such a multinational country as Russia. The research results will help to open a prospect in assessing the fundamental neurobiological aspects of developing Internet addiction and individualised therapeutic approach in Internet-dependent teenagers.

\section{Matirials and methods}

The overall prevalence assessment of Internet addiction in adolescents of 13-18 years old living in the city of Krasnoyarsk was carried out using the Chen Internet addiction Scale Test (CIAS, S.-H. Chen, 2003) in the adaptation of V. L. Malygin, K.A. Feklisov [5,6] in randomly selected secondary schools. 233 teenagers of Caucasian origin at the age of 13-18 years old were surveyed (average age is $15.3 \pm$ 1.6), with 111 girls and 122 boys being involved in the study. All examined ones or their parents gave written informed consent to participate. DNA was isolated using salting out method. Genotyping of rs1800497, rs4680 and rs2229910 was taken on 302 DNA samples of a population sampling of Caucasians in the Krasnoyarsk Territory using RT-PCR.

\section{Results and discussion}

An analysis of the content - structure of Internet addiction among adolescents in Krasnoyarsk resulted in the identification of three groups of adolescents: (1) adolescents with no Internet addiction behavior, a control group (the expected frequency was $60-75 \%$ ), the detected frequency being $80.3 \%$; (2) adolescents with a tendency for developing Internet addiction/pre-addictive stage (the expected frequency was $20-30 \%$ ), the detected frequency being $16.7 \%$; (3) adolescents with Internet addiction / behavior with an internet abuse component (the expected frequency was 5-10\%), the detected frequency being $3.0 \%$. As a result of the analysis, 7 Internet-dependent adolescents and 39 adolescents with a tendency to Internet-dependent behavior were identified, they were also paired (corresponding to gender, age and nationality) for sampling the biological material, further DNA extraction and genetic screening of polymorphic genes on IA susceptibility. According to the literature, a methodologically verified study of students in 9-11 grades in Moscow using the CIAS, i.e. questionnaire validated by the authors, showed that " $11.0 \%$ have some signs of Internet addiction, $42.0 \%$ abuse the Internet of all the adolescents examined $(\mathrm{n}=190) "$. 
Another study by the same research group demonstrated that " $4.25 \%$ were diagnosed as Internet addicts and $29.33 \%$ as Internet abusers of 1084 adolescents (the average age in the sampling was 15.6 years old)" [5].

We carried out a genotype test of the population sampling of Caucasians in Krasnoyarsk $(\mathrm{n}=302)$ rs1800497 DRD2, rs4680 COMT and rs2229910 NTRK3. The frequencies of genotype distribution were obtained: * CC 59.6\%,* CT $40.4 \%$, * TT 0\%; * AA 23.8\%, AG 52.4\%, * GG 23.8\%; * CC $37.4 \%$, * CG $46.0 \%$, * GG $16.6 \%$, respectively. The frequency of allelic variants of the studied SNPs corresponds to world data on Caucasoid populations (according to www.ensembl.org).

\section{Conclution}

As a result of increase in the group of teenagers, a comparative analysis of the distribution of polymorphic sites rs1800497, rs4680, rs2229910, rs25532 (serotonin transporter (SS-5HTTLPR) gene) and rs1044396 (nicotinic acetylcholine receptor subunit alpha 4 (CHRNA4) gene) is expected to carry out in adolescents of Central Siberia in order to identify candidate genes of developing Internet addiction.

\section{Acknowledgment}

The study was carried out with the financial support of the Russian Federal Property Fund in the framework of the scientific project No. 18-29-22032 \19.

\section{References}

[1] L. Cerniglia, F. Zoratto, S. Cimino, G. Laviola, M. Ammaniti, W. Adriani, "Internet Addiction in adolescence: Neurobiological, psychosocial and clinical issues," Neurosci Biobehav Rev, 2017, vol. 76, pp. 174-184.

[2] S. Park, J. Kim, C. Cho, "Prevalence of Internet addiction and correlations with family factors among South Korean adolescents", Adolescence, 2008, vol. 43(172), pp. 895-909

[3] D. Han, Y. Lee, K. Yang, E. Kim, I. Lyoo, P. Renshaw, "Dopamine genes and reward dependence in adolescents with excessive internet video game play", J Addict Med, 2007, vol. 1(3), pp. 133-138

[4] J. Jeong, J. Rhee, T. Kim, S. Kwak, S. Bang, H. Cho, Y. Cheon, J. Min, G. Yoo, K. Kim, J. Choi, S. Choi, D. Kim, "The association between the nicotinic acetylcholine receptor alpha4 subunit gene (CHRNA4) rs1044396 and Internet gaming disorder in Korean male adults", PLoS ONE, 2017, vol. 12(12), pp. e0188358.

[5] V. Malygin, Yu. Merkurieva, A. Iskandirova, E. Pakhtusova, A. Prokofieva, "Specific features of value orientations in adolescents with Internet addictive behaviour", Medical Psychology in Russia, 2015, vol. 33 (4), pp. 1-20.

[6] S.-H. Chen, L.-J. Weng, Y.-J. Su, H.-M. Wu, P.-F. Yang, "Development of a Chinese Internet Addiction Scale and Its Psychometric Study", Chinese Journal of Psychology, 2003, vol. 45(3), pp. 279-294. 